\title{
On the Estimation of Zero-Inefficiency Stochastic Frontier Models with Endogenous Regressors
}

\author{
Kien C. Tran* \\ Department of Economics \\ University of Lethbridge \\ 4401 University Drive W \\ Lethbridge, $\mathrm{AB}$ \\ T1K 3M4 Canada \\ and \\ Mike G. Tsionas \\ Department of Economics \\ Lancaster University Management School \\ LA1 4YX U.K \& \\ Athens University of Economics and Business, Greece
}

\begin{abstract}
In this paper, we investigate endogeneity issues in the zero-inefficiency stochastic frontier (ZISF) models by mean of simultaneous equation setting. Specifically, we allow for one or more regressors to be correlated with the statistical noise. A modified limited information maximum likelihood (LIML) approach is used to estimate the parameters of the model. Moreover, the firm specific inefficiency score is also provided. Limited Monte Carlo simulations show that the proposed estimators perform well in finite sample.

JEL Classification: C3, C5, C13.

Keywords: Endogeneity, Fully efficient firm, Limited information maximum likelihood, Firm specific inefficiency score, Zero-inefficiency stochastic frontier.

${ }^{*}$ Corresponding author. Tel.: +1 403329 2511; fax: +1 403329 2519. E-mail addresses: kien.tran@uleth.ca and

tsionas@oternet.gr.
\end{abstract}




\section{Introduction}

The so-called "zero-inefficiency stochastic frontier" (ZISF) model proposed by Kumbhakar, Parmeter and Tsionas (2012) and Rho and Schmidt (2015) allows for some firms in a typical sample to be fully efficient with a certain probability, a fact that we cannot preclude priori. Under standard assumptions on the composed errors, they suggest a maximum likelihood (ML) estimation procedure of the model's parameters as well as how to predict firm specific inefficiency. However, in their models, they assumed that all the regressors (or inputs, in the production frontier setting) are exogenous with respect to the statistical noise and inefficiency. In practice, this assumption might not be valid in some situations and consequently, invalidate the consistency of ML estimator and the estimates of firm specific inefficiency can be misleading. In this paper, we will relax this assumption and allow for one or more regressors to be correlated with the statistical noise in the composed error term; that is, we will investigate the case that one more of the regressors is endogenous, in the sense of simultaneous equation context.

In the standard stochastic frontier setting, the issues of endogeneity have recently been addressed by Amsler, Prokhorov and Schmidt (2016a, b), Tran and Tsionas (2013, 2105) and Kutlu (2010). However, to the best of our knowledge, it does not appear that the endogeneity problem has been considered in the ZISF setting. The plan of the paper is as follows. Section 2 introduces the ZISF model with endogeneity and discusses various assumptions as well as identification issues. Section 3 derives the limited information maximum likelihood (LIML) procedure as well as firm specific inefficiency predictor. Limited Monte Carlo simulations are presented in Section 4 to examine the finite sample performance of the proposed methods. Section 5 extends the model to allow for one or more inputs to be correlated with both statistical noise and inefficiency. Section 6 concludes the paper.

\section{The Model}

Consider the following Zero-Inefficiency Stochastic Frontier model with endogenous inputs:

$$
y_{i}=\left\{\begin{array}{ccc}
z_{1 i}^{\prime} \alpha+x_{i}^{\prime} \beta+v_{i} & \text { with probablity } & p\left(w_{i}\right) \\
z_{1 i}^{\prime} \alpha+x_{i}^{\prime} \beta+v_{i}-u_{i} & \text { with probablilty } & 1-p\left(w_{i}\right)
\end{array}\right.
$$

and

$$
x_{i}=Z_{2 i} \delta+e_{i},
$$

where $y_{i}$ is a scalar representing output of firm $i, z_{i}$ is a $q_{1} \times 1$ vector of exogenous inputs, $x_{i}$ is a $d \times 1$ vector of endogenous inputs, $v_{i}$ is random noise, $u_{i}$ is one-sided random variable representing technical inefficiency, $p($.$) is a known function representing the proportion of firms that are fully efficient and w_{i}$ is a $k \times 1$ vector of covariates which influence whether a firm is inefficient or not; $Z_{2 i}=I_{d} \otimes z_{2 i}^{\prime}$ where $z_{2 i}$ is a $q_{2} \times 1$ vector of exogenous instrument variables, and $e_{i}$ is a $d \times 1$ vector of two-sided random error terms which we assume that $e_{i} \sim N\left(0, \Omega_{e e}\right)$ where $\Omega_{e e}$ is a $d \times d$ covariance matrix. Following standard practice, we assume $v_{i} \sim N\left(0, \sigma_{v}^{2}\right), u_{i} \sim\left|N\left(0, \sigma_{u}^{2}\right)\right|, u_{i}$ is independent of $\eta_{i}=\left(v_{i}, e_{i}\right)^{\prime}$ and condition on $Z_{i}=\left(z_{1 i}, z_{2 i}\right)^{\prime}$, $\eta_{i} \sim N(0, \Omega)$ where $\Omega=\left[\begin{array}{cc}\sigma_{v}^{2} & \Omega_{v e} \\ \Omega_{e v} & \Omega_{e e}\end{array}\right]$, so that the endogeneity is due to the correlation between $v_{i}$ and $e_{i}$. For more general case where $e_{i}$ is allowed to be correlated with both $v_{i}$ and $u_{i}$, see Section 4 below. Also, to ensure that $p\left(w_{i}\right) \in[0,1]$, we assume $p\left(w_{i}\right)$ takes a logistic function, $p\left(w_{i}\right)=\frac{\exp \left(w_{i}^{\prime} \gamma\right)}{1+\exp \left(w_{i}^{\prime} \gamma\right)}$. Finally, for identification purpose, we assume that $\sigma_{u}^{2}>0$ and $q_{2} \geq d$ (so that there are at least as many instruments as $x$ 's).

\section{LIML Procedure}

To obtain the likelihood function, we follow density decomposition of Amsler, Prokhorov and Schmidt (2015a) approach, albeit one can also use Cholesky's decomposition approach as suggested in Kutlu (2010) and Tran and Tsionas (2013). Let $\varepsilon_{i}=v_{i}-u_{i}=y_{i}-z_{1 i}^{\prime} \alpha-x_{i}^{\prime} \beta, \tilde{\varepsilon}_{i}=\varepsilon_{i}-\mu_{c i}$ where $\mu_{c i}=\Omega_{v e} \Omega_{e e}^{-1} e_{i}$ with $e_{i}=x_{i}-Z_{i} \delta$ 
and $\sigma_{c}^{2}=\sigma_{v}^{2}-\Omega_{v e} \Omega_{e e}^{-1} \Omega_{e v}$. Next, since $u_{i}$ is independent of $v_{i}$ and $e_{i}$, we have:

$$
\begin{gathered}
f_{u, v, e}(u, v, e)=p(w) f_{v, e}(v, e)+\{1-p(w)\} f_{u}(u) f_{v, e}(v, e) \\
\quad=p(w) f_{v \mid e}(v) f_{e}(e)+\{1-p(w)\} f_{u}(u) f_{v \mid e}(v) f_{e}(e) \\
\quad=f_{e}(e)\left\{p(w) f_{v \mid e}(v)+(1-p(w)) f_{u}(u) f_{v \mid e}(v)\right\}
\end{gathered}
$$

where $f_{e}(e)=$ const $\times\left|\Omega_{e e}\right|^{-1} \times \exp \left(-\frac{1}{2} e^{\prime} \Omega_{e e}^{-1} e\right)$ and the distribution of $v \mid e$ is $N\left(\mu_{c}, \sigma_{c}^{2}\right)$. Consequently,

$$
f_{\varepsilon, e}(\varepsilon, e)=\int_{0}^{\infty} f_{u, v, e}(u, \varepsilon+u, e) d u=f_{e}(e) \int_{0}^{\infty} f_{v \mid e}(\varepsilon+u) d u .
$$

By making use of change in variables, $\tilde{\varepsilon}=\varepsilon-\mu_{c}$ and using the result in Aigner, Lovell and Schmidt (1977), the density of $\tilde{\varepsilon} \mid e$ can be shown to be

$$
f_{\tilde{\varepsilon} \mid e}(\tilde{\varepsilon})=\frac{2}{\sigma} \phi\left(\frac{\tilde{\varepsilon}}{\sigma}\right) \Phi\left(\frac{-\lambda \tilde{\varepsilon}}{\sigma}\right),
$$

where $\sigma^{2}=\sigma_{u}^{2}+\sigma_{c}^{2}=\sigma_{u}^{2}+\sigma_{v}^{2}-\Omega_{v e} \Omega_{e e}^{-1} \Omega_{e v}, \lambda=\frac{\sigma_{u}}{\sigma_{c}}$ and $\phi($.$) and \Phi($.$) are respectively the standard normal$ density and cdf. Thus, by writing $\varepsilon=\tilde{\varepsilon}+\mu_{c}$, we obtain:

$$
\begin{gathered}
f_{\varepsilon, e}(\varepsilon, e)=\left\{p(w)\left(2 \pi \sigma_{c}^{2}\right)^{-1 / 2} \exp \left(-\frac{1}{2 \sigma_{c}^{2}}\left(v-\mu_{c}\right)^{2}\right)+(1-p(w)) \sigma^{-1} \phi\left(\frac{\varepsilon-\mu_{c}}{\sigma_{c}}\right) \Phi\left(\frac{-\lambda\left(\varepsilon-\mu_{c}\right)}{\sigma_{c}}\right)\right\} \times \\
(2 \pi)^{-1} \Omega_{e e}^{-1 / 2} \exp \left(-\frac{1}{2} e^{\prime} \Omega_{e e}^{-1} e\right) .
\end{gathered}
$$

Then the log-likelihood function is:

$$
\ln L=\ln L_{1}+\ln L_{2}
$$

where

$$
\begin{array}{r}
\ln L_{1}=\sum_{i=1}^{n} \ln \left\{p\left(w_{i}\right)\left(2 \pi \sigma_{c}^{2}\right)^{-1 / 2} \exp \left(-\frac{1}{2 \sigma_{c}^{2}}\left(y_{i}-z_{1 i}^{\prime} \alpha-x_{i}^{\prime} \beta-\mu_{i c}\right)^{2}\right)+\right. \\
\left.\left[1-p\left(w_{i}\right)\right] \sigma^{-1} \phi\left(\frac{y_{i}-z_{1 i}^{\prime} \alpha-x_{i}^{\prime} \beta-\mu_{i c}}{\sigma_{c}}\right) \Phi\left(\frac{-\lambda\left(y_{i}-z_{1 i}^{\prime} \alpha-x_{i}^{\prime} \beta-\mu_{i c}\right)}{\sigma_{c}}\right)\right\}
\end{array}
$$

and

$$
\ln L_{2}=-\frac{n}{2} \ln \left|\Omega_{e e}\right|-\frac{1}{2} \sum_{i=1}^{n}\left(x_{i}-Z_{2 i} \delta\right)^{\prime} \Omega_{e e}^{-1}\left(x_{i}-Z_{2 i} \delta\right) .
$$

Note that, in a special case where $p()=0,.(7)$ reduces to the log-likelihood function of the standard SF models with endogenous regressors (e.g., Kutlu (2010), Tran and Tsionas (2013) and Amsler, Prokhorov and Schmidt (2016a)). On the other hand, when $p()=$.1 , it reduces to the log-likelihood function of simultaneous regression models. Finally, when there are no endogenous regressors, (i.e., $\Omega_{v e}=0$ ) it reduces to the case of ZISF models of Kumbhakar, Parmeter and Tsionas (2012) and Rho and Schmidt (2015).

Now by maximizing the log-likelihood function in (7) directly with respect to the parameters $\theta=$ $\left(\alpha, \beta, \sigma_{v}^{2}, \sigma_{u}^{2}, \delta, \Omega_{v e}, \Omega_{e e}, \gamma\right)$, we can obtain the LIML estimates. Or alternatively, we can use Generalized Method of Moment (GMM) approach of Tran and Tsionas (2013) which uses the moment conditions that are based on the score of the log-likelihood function. This GMM procedure is similar to the direct MLE. Finally, a control function type two-step procedure suggested by Kutlu (2010) can also be used. To construct a two-step procedure, let $\theta_{1}=\left(\alpha, \beta, \sigma_{v}^{2}, \sigma_{u}^{2}, \Omega_{v e}, \gamma\right)$ and $\theta_{2}=\left(\delta, \Omega_{e e}\right)$. Then in the first step, we maximize (9) with respect to $\theta_{2}$, and this is essentially the least square estimation of $x$ on $Z$ from the reduced form equations to obtain $\hat{\delta}$ and $\hat{\Omega}_{e e}=n^{-1} \sum_{i=1}^{n}\left(X_{i}-Z_{i}^{\prime} \hat{\delta}\right)\left(X_{i}-Z_{i}^{\prime} \hat{\delta}\right)^{\prime}$. In the second step, given the estimates of $\hat{\theta}_{2}$, we maximize (8) to obtain the remaining parameters $\theta_{1}$. Note that, unlike the direct MLE or GMM procedure, this two-step procedure is generally inefficient because it ignores the information about $\theta_{2}$ in (8) and treating as though it is known. Consequently, a practical implication is that the conventional estimated standard errors from step 2 are not correct, and they need to be adjusted to reflect the fact that $\theta_{2}$ have been 
estimated. One simple way to mitigate this problem is to use bootstrapping procedure. Or alternatively, one could follow Wooldridge (2010, Section 12.4.2) to construct the correct standard errors analytically.

Prediction of Firm Specific Inefficiency:

Once the parameters of the model have been estimated, we can use Jondrow et al. (1982) procedure to construct the estimate for $E\left(u_{i} \mid \varepsilon_{i}, e_{i}\right)$. Note that, we include $e_{i}$ as part of the conditional expectation because $e_{i}$ is informative about $u_{i}$ since $e_{i}$ is correlated with $v_{i}$, albeit $e_{i}$ is independent of $u_{i}$. Consequently, the conditional expectation $E\left(u_{i} \mid \varepsilon_{i}, e_{i}\right)$ can be rewritten as $E\left(u_{i} \mid \varepsilon_{i}, e_{i}\right)=E\left(u_{i} \mid \tilde{\varepsilon}_{i}, e_{i}\right)=E\left(u_{i} \mid \tilde{\varepsilon}_{i i}\right)$ where $\tilde{\varepsilon}_{i}$ is defined as before. Thus, it is straightforward to show that the conditional density of $u$ given $\tilde{\varepsilon}$ is

$$
f\left(u_{i} \mid \tilde{\varepsilon}_{i}\right)=\left\{\begin{array}{cc}
0 & \text { with probability } \quad p\left(w_{i}\right) \\
N_{+}\left(\mu_{* i}, \sigma_{*}^{2}\right) & \text { with probability }\left(1-p\left(w_{i}\right)\right)
\end{array},\right.
$$

where $N_{+}($.$) denotes the truncated normal, \mu_{* i}=-\tilde{\varepsilon}_{i} \sigma_{u}^{2} / \sigma^{2}$ and $\sigma_{*}^{2}=\sigma_{u}^{2} \sigma_{c}^{2} / \sigma^{2}$ where $\sigma^{2}$ and $\sigma_{c}^{2}$ are defined previously. Then, the conditional mean of $u_{i}$ given $\tilde{\varepsilon}_{i}$ is:

$$
E\left(u_{i} \mid \tilde{\varepsilon}_{i}\right)=\left(1-p\left(w_{i}\right)\right) \sigma_{*}\left[\frac{\phi\left(\lambda \tilde{\varepsilon}_{i} / \sigma\right)}{1-\Phi\left(\lambda \tilde{\varepsilon}_{i} / \sigma\right)}-\frac{\lambda \tilde{\varepsilon}_{i}}{\sigma}\right],
$$

A point estimator of individual specific inefficiency score $\hat{u}_{i}=\hat{E}\left(u_{i} \mid \tilde{\varepsilon}_{i}\right)$ can be obtained by replacing the unknown parameters in (10) by their estimates using one of the approaches discussed above and $\tilde{\varepsilon}_{i}$ by $\hat{\tilde{\varepsilon}}_{i}$. In addition, one can also construct the posterior estimates of inefficiency score $\tilde{u}_{i}$ by calculating $\tilde{u}_{i}=$ $\left(1-\hat{\pi}\left(w_{i}\right)\right) \hat{u}_{i}$ where

$$
\hat{\pi}\left(w_{i}\right)=\frac{\left(\hat{p}\left(w_{i}\right) / \hat{\sigma}_{v}\right) \phi\left(\hat{\tilde{\varepsilon}}_{i} \hat{\lambda} / \hat{\sigma}_{v}\right)}{\left(\hat{p}\left(w_{i}\right) / \hat{\sigma}_{v}\right) \phi\left(\tilde{\tilde{\varepsilon}}_{i} \hat{\lambda} / \hat{\sigma}_{v}\right)+\left(1-\hat{p}\left(w_{i}\right)\right)(2 / \hat{\sigma}) \phi\left(\hat{\tilde{\varepsilon}}_{i} \hat{\lambda} / \hat{\sigma}\right) \Phi\left(-\hat{\tilde{\varepsilon}}_{i} \hat{\lambda} / \hat{\sigma}\right)},
$$

is the posterior estimate of the probability of being fully efficient.

We make the following remarks. First, it is important to recognize that the validity of the above proposed procedures hinges upon the conditions that both the frontier function and the reduced form are correctly specified as discussed in Amsler, Prokhorov and Schmidt (2016a). Second, the above procedure can be easily modified to accommodate other exogenous environmental variables that appear in the distribution of $u_{i}$ without conceptual estimation issues. Finally, a practical implication of the proposed approach is that one can easily test the null hypothesis of exogeneity of $x_{i}$ (i.e., $H_{0}: \Omega_{v e}=0$ ) using standard likelihood ratio statistics.

\section{Monte Carlo Simulations}

In this section, we conduct some simulations to examine the finite sample performance of the proposed estimators. To this end, we consider the following data generating process (DGP):

$$
\begin{aligned}
& y_{i}=\left\{\begin{array}{ccc}
1+\alpha z_{1 i}+\beta x_{i}+v_{i} & \text { with probablity } & p\left(w_{i}\right) \\
1+\alpha z_{1 i}+\beta x_{i}+v_{i}-u_{i} & \text { with probablilty } & 1-p\left(w_{i}\right)
\end{array},\right. \\
& x_{i}=1+\delta z_{2 i}+\varepsilon_{i}, \quad \varepsilon_{i} \sim N\left(0, \sigma_{\varepsilon}^{2}\right) \\
& p\left(w_{i}\right)=\frac{\exp \left(\gamma w_{i}\right)}{1+\exp \left(\gamma w_{i}\right)},
\end{aligned}
$$

where the one-sided error $u_{i}$ is generated as $\left|N\left(0, \sigma_{u}^{2}\right)\right|$ and the vector of random variables $\left(z_{1 i}, z_{2 i}, w_{i}\right)^{\prime}$ is generated as $N\left(0, I_{3}\right)$. The vector of random errors, $\left(v_{i}, \varepsilon_{i}\right)^{\prime}$ is generated from $N\left(0_{2}, \Omega\right)$ where $\Omega=$ $\left(\begin{array}{cc}\sigma_{v}^{2} & \rho \sigma_{v} \sigma_{\varepsilon} \\ \rho \sigma_{v} \sigma_{\varepsilon} & \sigma_{\varepsilon}^{2}\end{array}\right)$ For all of our simulations, we set $\{\alpha, \beta, \delta, \gamma\}=[1.0,1.0,0.75,0.5\}, \sigma_{v}^{2}=\sigma_{\varepsilon}^{2}=1, \sigma_{u} \in$ $\{1.0,2.5,5.0\}$ and $\rho=\{0.0,0.5,0.8\}$ which corresponds to strong heterogeneity. We consider $n=2000$ and for each experimental design and 5,000 replications are performed. We report the root means square errors (RMSE) of three estimators: direct MLE, GMM and two-step MLE. The results are displayed in Table 1. For conservation of space, we only report the results for the case where $\rho=0.8$. The results for other cases 
are very similar and they are available from the authors upon request. Overall, our simulation results show that all three procedures are performed very well in finite sample regardless the values of $\rho$ and $\sigma_{u}^{2}$. Also, as expected, the RMSEs of the direct MLE and GMM estimators are very similar and that the RMSEs of the two-step estimator are slightly higher in almost all cases. 
Table 1. RMSE of Direct MLE, GMM and Two-Step MLE

\begin{tabular}{|c|c|c|c|c|}
\hline & $\alpha$ & $\beta$ & $\gamma$ & $\delta$ \\
\hline \multicolumn{5}{|l|}{$\rho=0.8, \sigma_{u}=1$} \\
\hline Direct MLE & 0.008 & 0.007 & 0.008 & 0.005 \\
\hline GMM & 0.009 & 0.008 & 0.009 & 0.007 \\
\hline Two-step MLE & 0.014 & 0.016 & 0.015 & 0.015 \\
\hline \multicolumn{5}{|l|}{$\rho=0.8, \sigma_{u}=2.5$} \\
\hline Direct MLE & 0.005 & 0.004 & 0.004 & 0.004 \\
\hline GMM & 0.007 & 0.005 & 0.006 & 0.006 \\
\hline Two-step MLE & 0.012 & 0.013 & 0.011 & 0.010 \\
\hline \multicolumn{5}{|l|}{$\rho=0.8, \sigma_{u}=5$} \\
\hline Direct MLE & 0.004 & 0.003 & 0.003 & 0.004 \\
\hline GMM & 0.005 & 0.003 & 0.003 & 0.005 \\
\hline Two-step MLE & 0.010 & 0.008 & 0.009 & 0.008 \\
\hline
\end{tabular}

\section{Extension: $e_{i}, v_{i}$ and $u_{i}$ are all potentially correlated}

In this section, we consider the more general case where we allow for $e_{i}, v_{i}$ and $u_{i}$ are all potentially correlated since in practice, it is unknown (and cannot be tested) whether endogeneity is caused by the correlation with the statistical noise or the inefficiency term ${ }^{1}$. First, rewrite (4) as:

$$
\begin{gathered}
f_{u, v, e}(u, v, e)=p(w) f_{v, e}(v, e)+\{1-p(w)\} f_{u, v, e}(u, v, e) \\
=p(w) f_{v \mid e}(v) f_{e}(e)+\{1-p(w)\} f_{u, v, e}(u, \varepsilon+u, e),
\end{gathered}
$$

and from (11) it is clear that we need to determine a joint distribution $f_{u, v, e}(u, v, e)$ in such way that the marginal distributions of $u_{i}$ and $\eta_{i}=\left(v_{i}, e_{i}^{\prime}\right)^{\prime}$ are half-normal and multivariate normal, respectively, and $u_{i}$ and $\eta_{i}$ are correlated in some sensible manners. One possible and convenient method is to use Copula approach to model the dependency between $u_{i}$ and $\eta_{i}$ as suggested in Amsler, Prokhorov and Schmidt (2016a). Let $h\left(u_{i}, \eta_{i}\right)$ denote the joint density of $\left(u_{i}, \eta_{i}^{\prime}\right)^{\prime}$, and suppose that the marginal density and cdf of $u_{i}$ and $\eta_{i}$ are $f\left(u_{i}\right), f\left(\eta_{i}\right)$ and $F\left(u_{i}\right), F\left(\eta_{i}\right)$, respectively. Then by Sklar's Theorem (see Nelsen (2016)), we can write:

$$
h\left(u_{i}, \eta_{i}\right)=c\left(F\left(u_{i}\right), F\left(\eta_{i}\right)\right) \cdot f\left(u_{i}\right) f\left(\eta_{i}\right)
$$

where $c(.,$.$) is the copula density and its arguments are uniform random variables. Thus from (12), it is clear$ that the joint distribution $h(.,$.$) can be determined if we specify the marginal densities and the copula c(.,$.$) .$ Under our maintained assumptions of half-normal marginal for $u_{i}$ and normal marginal for the elements of $\eta_{i}=\left(v_{i}, e_{i}^{\prime}\right)^{\prime}$, then an obvious and convenient choice for the copula is Gaussian copula because it implies that the distribution of $\eta_{i}$ is multivariate normal which is what we want to achieve. Under these specifications, the joint density of $h\left(u_{i}, \eta_{i}\right)$ can be obtained from (12), and let $\tilde{f}_{u, v, e}(u, \varepsilon+u, e)$ denote this joint density. To derive the likelihood function, we need the joint density of $\varepsilon_{i}$ and $e_{i}$ so we need to integrate out $u_{i}$ from $f_{u, v, e}(u, v, e)$ in (11). However, this integral may not be analytically tractable, and one possible solution is

\footnotetext{
${ }^{1}$ We would like to thank an anonymous referee for suggesting this extension.
} 
to evaluate it by numerical method. To do this, integrating out $u_{i}$ from (11) yields

$$
\begin{aligned}
f_{\varepsilon, e}(\varepsilon, e) & =\int_{0}^{\infty}\left[p(w) f_{v \mid e}(v) f_{e}(e)+\{1-p(w)\} \tilde{f}_{u, v, e}(u, \varepsilon+u, e)\right] d u \\
= & \int_{0}^{\infty}\left[\frac{p(w) f_{v \mid e}(v) f_{e}(e)+\{1-p(w)\} \tilde{f}_{u, v, e}(u, \varepsilon+u, e)}{f_{u}(u)}\right] f_{u}(u) d u \\
= & E_{u}\left[\frac{p(w) f_{v \mid e}(v) f_{e}(e)}{f_{u}(u)}\right]+E_{u}\left[\frac{\{1-p(w)\} \tilde{f}_{u, v, e}(u, \varepsilon+u, e)}{f_{u}(u)}\right]
\end{aligned}
$$

where $E_{u}$ denotes the expectation over the distribution of $u$. Thus, we can approximate (13) by drawing $u_{s}, s=1, \ldots, S$ from the distribution of $u$ and taking average over these draws to give the simulated value of $f_{\varepsilon, e}(\varepsilon, e)$ :

$$
\hat{f}_{\varepsilon, e}(\varepsilon, e)=\frac{1}{S} \sum_{s=1}^{S}\left[\frac{p(w) f_{v \mid e}(v) f_{e}(e)}{f_{u}\left(u_{s}\right)}\right]+\frac{1}{S} \sum_{s=1}^{S}\left[\frac{\{1-p(w)\} \tilde{f}_{u, v, e}\left(u, \varepsilon+u_{s}, e\right)}{f_{u}\left(u_{s}\right)}\right]
$$

and the simulated log-likelihood is given by:

$$
\ln \hat{L}=\sum_{i=1}^{n} \ln \hat{f}_{\varepsilon, e}\left(\varepsilon_{i}, e_{i}\right)
$$

The simulated maximum likelihood estimator (SMLE) can be obtained by maximizing (15) with respect to all the parameters including those that appear in the copula. Under certain regularity conditions, standard results of Gourieroux and Monfort (1993) show that the SMLE is consistent and has the same asymptotic distribution as MLE based on $f_{\varepsilon, e}\left(\varepsilon_{i}, e_{i}\right)$ provided that $n \rightarrow \infty, S \rightarrow \infty$ and $\frac{S}{\sqrt{n}} \rightarrow \infty$. Finally, the inefficiency term $\tilde{u}_{i}=E\left(u_{i} \mid \varepsilon_{i}, e_{i}\right)$ can be predicted using similar approach as in Amsler, Prokhorov and Schmidt (2014).

Last but not least, another possible extension is to also allow for one or more covariates $w_{i}$ in the probability of efficient firm and all of other environmental variables (if any) to be correlated with both the statistical noise and inefficiency term, and this case would provide the most general framework of the model. However, identification of the model may be an issue and it is beyond the scope of this paper, and we will leave it for future research.

\section{Conclusion}

In this paper, we investigate the endogeneity issues in the ZISF framework by mean of simultaneous equation setting. Specifically, we allow for one or more regressors to be correlated with the statistical noise in the composed error. We modify the LIML procedure to estimate all the parameters of the model and construct the prediction of firm specific inefficiency score. Small Monte Carlo simulations reveal that the proposed estimators perform well in finite sample in term of RMSE. We also extend the model to allow for all potential correlations among the errors and suggest a simulated maximum likelihood procedure to estimate the model parameters as well as the inefficiency scores.

\section{Acknowledgments}

We would like to thank an anonymous referee for useful comments and suggestions that led to substantial improvement of the paper.

\section{References}

Amsler, C., Prokhorov, A., Schmidt, P., 2014. Using copulas to model time dependence in stochastic frontier models. Econometric Reviews, 33, 497-522.

Amsler, C., Prokhorov, A., Schmidt, P., 2016a. Endogeneity in stochastic frontier models. Journal of Econometrics, 190, 280-288. 
Amsler, C., Prokhorov, A., Schmidt, P., 2016b. Endogeneity environmental variables in stochastic frontier models. Working paper presented at the XI North American Productivity Workshop, June 15-18, 2016, Quebec City, Quebec, Canada.

Gourieroux, C., Monfort, A., 1993. Simulation-based inference: A survey with special reference to panel data models. Journal of Econometrics, 59, 5-33.

Jondrow, J., Lovell, C.A.K., Materov, I.S., \& Schmidt P. 1982. On the estimation of technical inefficiency in the stochastic frontier production function model. Journal of Econometrics, 19 (2/3), 233-238.

Kumbhakar, S., Parmeter, C.F., \& Tsionas, E.G. 2013. A zero inefficiency stochastic frontier model. Journal of Econometrics, 172, 66-76.

Kutlu, L. 2010. Battese-Coelli estimator with endogenous regressors. Economics Letters, 109, 79-81.

Nelsen, R.B. 2006. An Introduction to Copulas. In: Springer Series in Statistics, vol.139. Springer.

Rho, S., \& Schmidt, P. 2015. Are all firms inefficient? Journal of Productivity Analysis, 43, 327-349.

Tran, K.C. and Tsionas, E.G. 2015. Endogeneity in stochastic frontier models: copula approach without external instruments. Economics Letters, 133, 85-88.

Tran, K.C., Tsionas, E.G. 2013. GMM estimation of stochastic frontier models with endogenous regressors. Economics Letters, 118, 233-236.

Wooldridge, J.M. 2010, Econometric Analysis of Cross Section and Panel Data. MIT Press. 